\title{
Texture Development during Grain Growth in Nonoriented Electrical Steels
}

\author{
Jong-Tae PARK ${ }^{1,2)}$ and Jerzy A. SZPUNAR ${ }^{2)}$ \\ 1) Technical Research Laboratories, POSCO, Pohang-shi, Kyungbuk, Korea. E-mail: jtpark20@posco.co.kr \\ 2) Department of Mining, Metals and Materials Engineering, McGill University, 3610 University Street, Montreal, Quebec, \\ H3A 2B2 Canada.
}

(Received on November 4, 2004; accepted on January 26, 2005)

\begin{abstract}
Nonoriented electrical steels should have both low core loss and high permeability. These magnetic properties are largely affected by grain size and texture. The research on grain size optimization during grain growth has been very extensive whereas little attention has been paid to texture transformation during grain growth. In the present study, based on obtained experimental results, a mechanism of texture development during grain growth in nonoriented electrical steels is proposed. In the $2 \%$ Si specimens, the major texture components, Goss and $\{111\}\langle 112\rangle$ components, are weakened during grain growth. In the $1 \%$ Si specimens, the main texture components, $\{111\}\langle 112\rangle$ and $\{111\}\langle 110\rangle$, are strengthened. It is proposed that for a texture component to be continuously strengthened during grain growth, the grains of the specific orientation should have not only a size advantage over those of other orientations, but also a higher frequency of high angle, high energy grain boundaries.
\end{abstract}

KEY WORDS: nonoriented electrical steel; grain growth; texture; misorientation angle; grain size.

\section{Introduction}

Nonoriented electrical steels should have both low core loss and high permeability. These magnetic properties are influenced by grain size as well as texture. As grain size increases, hysteresis loss decreases whereas eddy current loss increases. As a result, there is an optimum grain size which minimizes the sum of hysteresis loss and eddy current loss. ${ }^{1)}$ In addition, the optimum grain size changes with chemical composition and texture. ${ }^{2,3)}$ For example, the optimum grain sizes are 100 and $150 \mu \mathrm{m}$ for $1.85 \%$ and $3.2 \%$ Si steel, respectively. On the other hand, texture also strongly influences hysteresis loss. Park et al. ${ }^{3)}$ reported that materials with a texture favorable for magnetic properties show lower core loss than those with an unfavorable texture, although they have almost the same grain size. This indicates that the core loss can be further decreased by texture control. In nonoriented electrical steels, research on grain size during grain growth has been extensively carried out $^{3-5)}$ while little attention has been paid to texture evolution during grain growth.

The increase in grain size during grain growth is often associated with changes in texture. Grain growth is also related to the migration of grain boundaries. Experimental results on texture evolution during grain growth have so far been very scarce and even contradictory. In the case of low carbon steels, two fairly conflicting results concerning the effect of grain growth on texture development were published. ${ }^{6,7)}$ Hutchinson et al. ${ }^{6)}$ reported that the $\{111\}$ texture component during grain growth is strengthened. In contrast,
Balke $^{7)}$ found that $\gamma$-fibre components, such as $\{111\}\langle 112\rangle$ and $\{111\}\langle 110\rangle$, are simultaneously reduced in strength during grain growth. In addition, since the orientation dependence of grain boundary energy and boundary mobility has not yet been fully understood, the mechanism for texture development during grain growth even in low carbon steels remains unclear. Nonoriented electrical steels undergo or do not undergo $\alpha-\gamma$ phase transformation during hot rolling according to silicon content. In general, there is a $\alpha-\gamma$ phase transformation in $1 \%$ silicon steels whereas there is no phase transformation in $2 \%$ silicon steels. The phase transformation makes a big difference in the microstructure prior to cold rolling. In our previous research, ${ }^{8)}$ it has been found that the recrystallization textures in $1 \%$ and $2 \%$ Si specimens are quite different due to their different microstructures prior to cold rolling. Therefore, texture change during the following grain growth stage needs to be investigated in $1 \%$ and $2 \% \mathrm{Si}$ specimens annealed between $740^{\circ} \mathrm{C}$ and $950^{\circ} \mathrm{C}$. Furthermore, in order to propose the mechanism of texture development during grain growth in nonoriented electrical steel, the misorientation angle distribution with adjacent grains and the average size of grains of specific orientations are measured using EBSD (Electron Backscatter Diffraction) techniques.

\section{Experimental Procedure}

The starting materials were two kinds of hot bands that have the chemical compositions shown in Table 1. The hot bands containing $2 \% \mathrm{Si}$ were annealed at $1000^{\circ} \mathrm{C}$ for $5 \mathrm{~min}$ 
to obtain a coarse initial grain size prior to cold rolling. The initial average grain sizes prior to cold rolling were $40 \mu \mathrm{m}$ for $1 \% \mathrm{Si}$ and $115 \mu \mathrm{m}$ for $2 \% \mathrm{Si}$. The specimens with different initial grain sizes were cold rolled to $0.5 \mathrm{~mm}$ by $75 \%$ reduction using a laboratory mill. After that, cold rolled specimens with $1 \% \mathrm{Si}$ were annealed for $5 \mathrm{~min}$ at temperatures between $740^{\circ} \mathrm{C}$ and $900^{\circ} \mathrm{C}$ to promote grain growth. For cold rolled specimens with $2 \% \mathrm{Si}$, annealing was performed at temperatures between $790^{\circ} \mathrm{C}$ and $950^{\circ} \mathrm{C}$ for the same duration. All heat treatments were carried out in an Ar atmosphere to avoid oxidation. The microstructures of the specimens annealed at different temperatures were observed on the cross sectional area, parallel to the rolling direction. A nital solution of $2 \%$ was used as an etchant.

The X-ray texture of the specimens investigated was measured with Mo radiation using a Siemens D-500 X-ray diffractometer. The three incomplete pole figures of $\{110\}$, $\{200\}$ and $\{211\}$ were measured at different layers, defined as parameter $S=2 a / d$, where $a$ represents the distance from the center layer of the specimen and $d$ the thickness of the specimen. $S=0$ corresponds to the center and $S=1$ to the surface layer. For pole figure measurements, the specimens were mechanically polished and then electropolished in a solution of $800 \mathrm{~mL}$ glacial acetic acid and $200 \mathrm{~mL}$ perchloric acid $\left(\mathrm{HClO}_{4}\right)$, in order to remove the surface layer that was deformed during previous mechanical polishing. The orientation distribution function (ODF) was calculated from the three pole figures using TexTools, a texture analysis software package from ResMat.Corp.

EBSD measurements were performed using OIM (Orientation Imaging Microscopy) developed by TexSEM Laboratories, linked with a Phillips XL 30 FEG SEM. The

Table 1. Chemical compositions of specimens used in this study $(w t \%)$.

\begin{tabular}{|c|c|c|c|c|c|c|}
\hline $\mathrm{C}$ & $\mathrm{Si}$ & $\mathrm{Al}$ & $\mathrm{Mn}$ & $\mathrm{P}$ & $\mathrm{S}$ & $\mathrm{N}$ \\
\hline 0.0050 & 1.0 & 0.27 & 0.26 & 0.0026 & 0.0050 & 0.0025 \\
\hline 0.0035 & 2.0 & 0.31 & 0.24 & 0.0018 & 0.0030 & 0.0020 \\
\hline
\end{tabular}

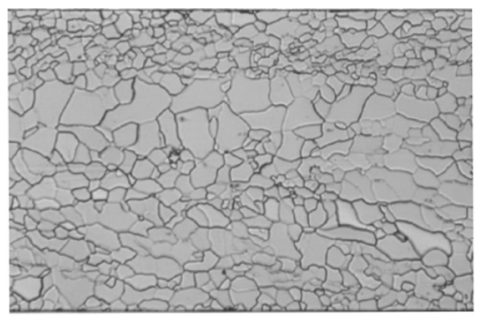

$790^{\circ} \mathrm{C}$

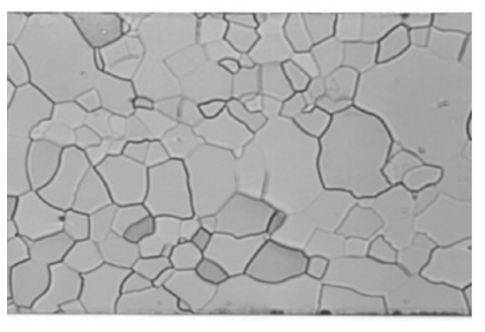

$900^{\circ} \mathrm{C}$ measurements were made on the cross sections defined by the rolling and normal directions. For analysis, grain orientations were grouped into 7 components: $\{001\}\langle 110\rangle$, $\{112\}\langle 110\rangle, \quad\{111\}\langle 110\rangle, \quad\{110\}\langle 110\rangle, \quad\{111\}\langle 112\rangle$, $\{011\}\langle 100\rangle$ (Goss) and $\{100\}\langle 001\rangle$ (Cube). The other texture components are considered to represent a random orientation. To define a particular texture component a deviation angle of $20^{\circ}$ was applied. In other words, if a grain is within $20^{\circ}$ from an ideal orientation, the grain belongs to the ideal orientation. However, no overlap between two arbitrary orientations is possible, because a grain belongs to the ideal orientation to which it is the closest. The average diameter and the misorientation angle distribution of grains of specific orientations were calculated using OIM software provided by TexSEM Laboratories. In addition, the area fraction of various texture components was calculated to investigate the change of various texture components during grain growth.

\section{Results and Discussion}

\subsection{Texture Development in $2 \%$ Si Steels}

Figure 1 shows the change of optical microstructures at annealing temperatures in the $2 \% \mathrm{Si}$ specimens. As the annealing temperature increases, the grain size on average increases. In the specimen annealed at $790^{\circ} \mathrm{C}$, the microstructure has an inhomogeneous grain size distribution throughout the thickness of the specimen. Grain size is generally bigger near the center layer and smaller near the surface layer. However, as the annealing temperature increases further, the inhomogeneous grain size distribution throughout the thickness of the specimen tends to disappear. The different grain sizes throughout the thickness of the specimen may be related to different textures. In order to examine this possibility, the X-ray texture was measured at different layers. Figure 2 shows the $\varphi_{2}=45^{\circ}$ section of ODF for the $2 \%$ $\mathrm{Si}$ specimen annealed at $790^{\circ} \mathrm{C}$. It is apparent that even after recrystallization the textures at different thickness layers are still quite different. They have not only different dominant texture components, but also different intensities.

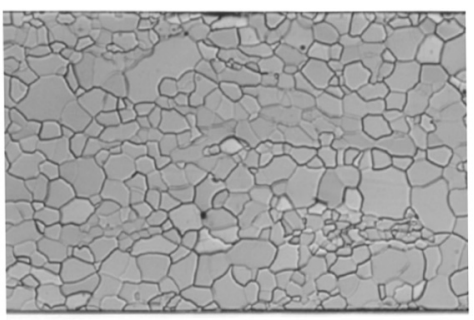

$850^{\circ} \mathrm{C}$

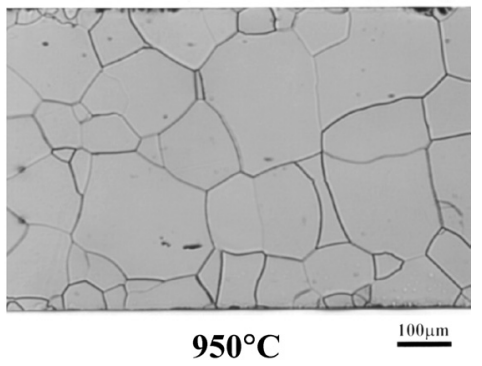

Fig. 1. Optical microstructures at annealing temperature in the $2 \% \mathrm{Si}$ specimens. 


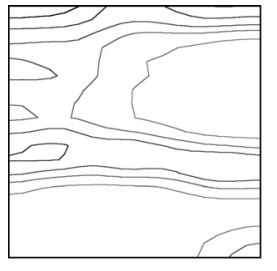

(a) $\mathrm{S}=\mathbf{0 . 9 5}$
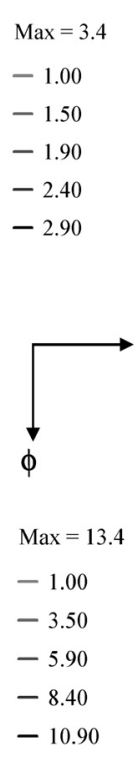

(c) $\mathrm{S}=\mathbf{0 . 5}$

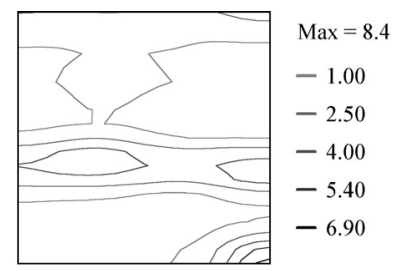

(b) $\mathrm{S}=\mathbf{0 . 7 5}$

$\varphi 1$

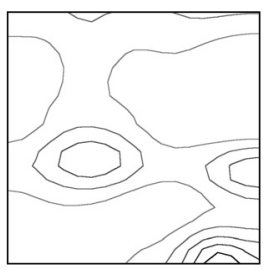

(d) $\mathrm{S}=\mathbf{0}$
Fig. 2. $\varphi_{2}=45^{\circ}$ sections of ODF at different layers in the $2 \% \mathrm{Si}$ specimen annealed at $790^{\circ} \mathrm{C}$.

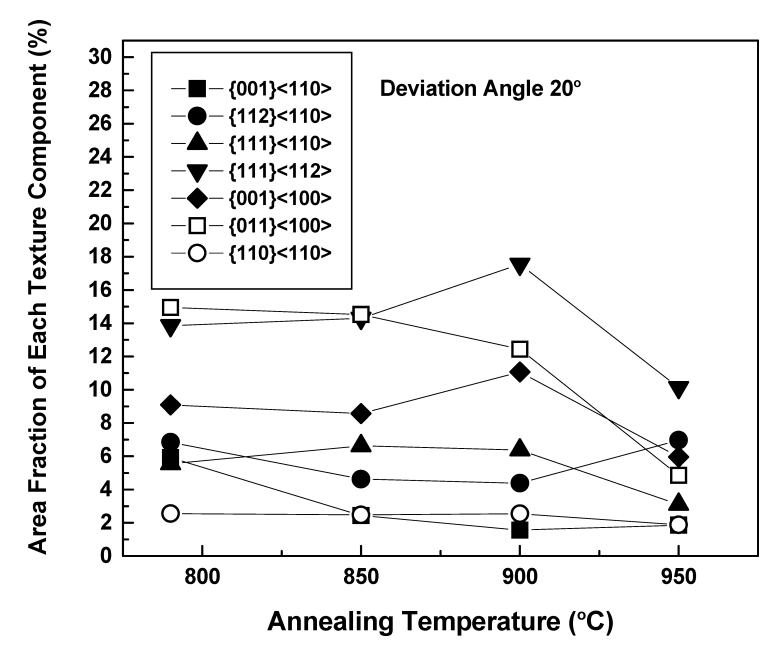

Fig. 3. Area fraction of grains with various orientations with annealing temperature in the $2 \% \mathrm{Si}$ specimens.

At the layers of $S=0.75$ through $S=0$, the dominant texture is the Goss component, although the densities of Goss components are quite different. At the center layer, $\{110\}\langle 115\rangle$ component, $15^{\circ}$ away from ideal Goss orientation, is dominant. One can see from Figs. 1 and 2 that grains of different sizes are associated with different orientations. This relationship between grain size and orientation was investigated using EBSD measurements and will be discussed later.

$2 \% \mathrm{Si}$ specimens have strong texture inhomogeneity throughout the thickness of the specimen even during the grain growth process. Accordingly, it is reasonable to measure texture on a cross section in order to investigate texture development during grain growth. Figure 3 represents the changes in the area fraction of various texture components obtained from EBSD measurements with annealing temperature. Two competing texture components during grain growth are Goss and $\{111\}\langle 112\rangle$. The area fraction of the Goss component continues to decrease with annealing temperature. On the other hand, the area fraction of the

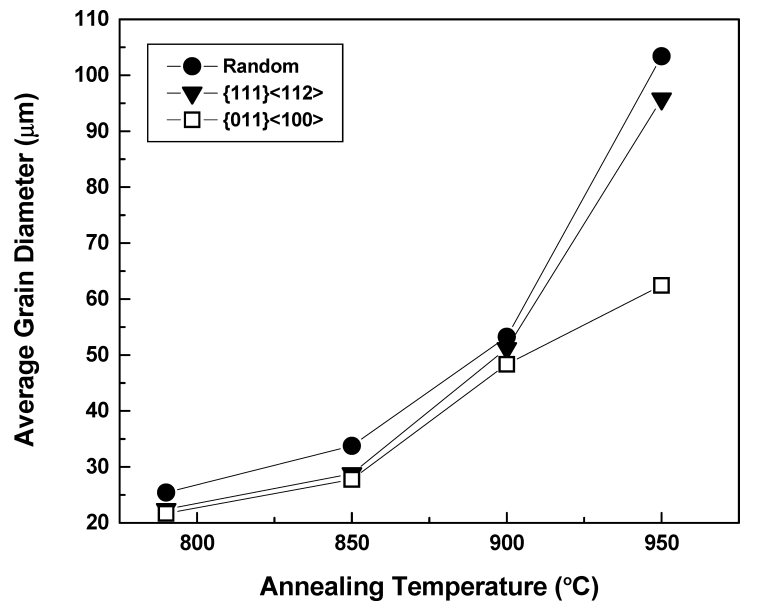

Fig. 4. Average grain diameter of grains with various orientations with annealing temperature in the $2 \% \mathrm{Si}$ specimens.

$\{111\}\langle 112\rangle$ texture component increases up to $900^{\circ} \mathrm{C}$ and then decreases with annealing temperature. It should be noted in Fig. 3 that the area fraction of all texture components except the $\{112\}\langle 110\rangle$ component decreases at the annealing temperature of $950^{\circ} \mathrm{C}$, so that the texture becomes more random. However, the intensity of the $\{112\}\langle 110\rangle$ texture component does not change significantly in the annealing temperature range investigated.

The average diameters of grains of specific orientations were calculated using OIM software provided by TexSEM Laboratories. The total number of grains used for this calculation were as follows: 2663 grains at $790^{\circ} \mathrm{C}, 1144$ grains at $850^{\circ} \mathrm{C}, 1037$ grains at $900^{\circ} \mathrm{C}$ and 954 grains at $950^{\circ} \mathrm{C}$. Figure 4 shows the change of average diameters of grains having Goss, $\{111\}\langle 112\rangle$ and random orientation with annealing temperature. Average grain diameter is quite different depending on the orientation of grains, especially at $950^{\circ} \mathrm{C}$. The average diameter of grains of $\{111\}\langle 112\rangle$ orientation is bigger than that of Goss orientation, but smaller than that of random orientation for all annealing temperatures investigated. Different sizes of grains of specific orientations may arise from the different growth rates for different orientations. We may expect that the growth rate of $\{111\}\langle 112\rangle$ grains is higher than that of grains with Goss orientation.

In order to investigate the reason why grains of Goss and $\{111\}\langle 112\rangle$ orientations have different grain sizes, grain boundary misorientation for grains of these orientations was counted from EBSD measurements. The number of grain boundaries used for the calculation was 650 and 512 for Goss and $\{111\}\langle 112\rangle$ orientations, respectively. Figure 5 shows misorientation angle distribution for both texture components in the specimen annealed at $850^{\circ} \mathrm{C}$. Goss grains have a lot of low angle grain boundaries (less than $15^{\circ}$ misorientation). The frequency of these low angle grain boundaries is around $45 \%$, which means that the Goss grains have grains of similar orientation as their neighbors. On the other hand, $\{111\}\langle 112\rangle$ grains show a very different grain boundary character distribution when compared with Goss grains. Each grain boundary frequency, in any given misorientation range, changes between $10 \%$ and $25 \%$. This indicates that the $\{111\}\langle 112\rangle$ grains are relatively uniformly distributed. Consequently, $\{111\}\langle 112\rangle$ grains have a 


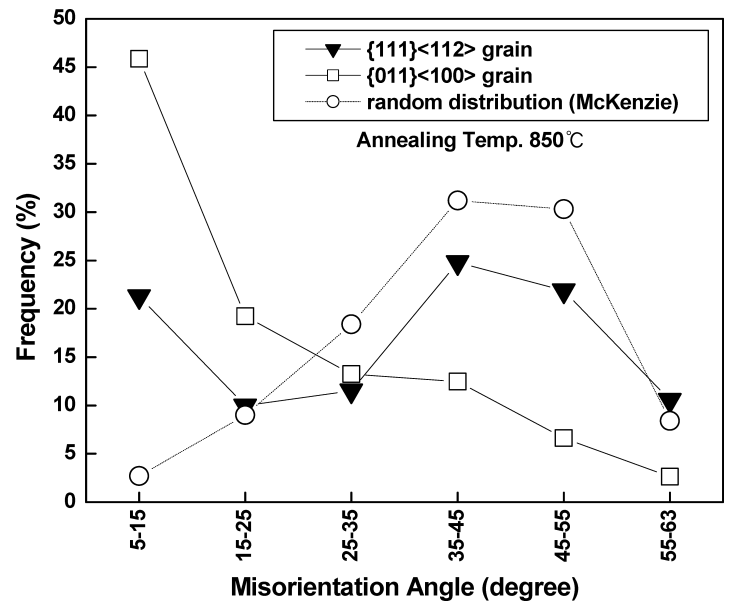

Fig. 5. Correlated misorientation angle distribution of grains with $\{011\}\langle 100\rangle$ and $\{111\}\langle 112\rangle$ orientation in the $2 \% \mathrm{Si}$ specimen annealed at $850^{\circ} \mathrm{C}$.

much higher frequency of high angle grain boundaries than Goss grains. An EBSD map coded with crystal orientation in the specimen annealed at $850^{\circ} \mathrm{C}$ is shown in Fig. 6. Here, dark yellow grains represent Goss orientation and blue grains represent $\{111\}\langle 112\rangle$. White grains are considered as having random orientation. Grains of Goss orientation within the circles in the microstructure have a smaller size than those of other orientations. The Goss grains form colonies, which indicates that the Goss grains have a similar orientation to each other. As a result, the Goss grains have a higher frequency of low angle grain boundaries. In general, the velocity of grain boundaries is expressed as the product of mobility and driving pressure. However, since the driving pressure for grain growth is extremely low, ${ }^{9}$ the mobility of grain boundaries may be a major factor in controlling the velocity of grain boundaries. The grains of Goss orientation have many low mobility, low angle grain boundaries and hence the growth rate of these grains must be low since the mobility of high angle grain boundaries is significantly higher than that of low angle grain boundaries. ${ }^{10-12)}$ In addition, it is generally accepted that grains with high mobility, high angle grain boundaries are expected to grow during grain growth, whereas grains surrounded by low angle grain boundaries are not expected to continue to grow. Therefore, the Goss grains have a high probability of being consumed by grains having other orientations, usually a random orientation. From Figs. 3 and 5, one can see that although the $\{111\}\langle 112\rangle$ grains have more favorable grain boundaries for growth than the Goss grains, they will also be ultimately consumed by grains of random orientation. In summary, even though the Goss and $\{111\}\langle 112\rangle$ texture components are the two strongest components both after recrystallization and at the beginning of the grain growth stage, the grains of these orientations are eventually consumed by grains of random orientation during the following stages of grain growth.

\subsection{Texture Development in $1 \%$ Si Steels}

Figure 7 illustrates the change of optical microstructures with annealing temperature in the $1 \% \mathrm{Si}$ specimens. The grain size, on average, increases as the annealing temperature increases. Unlike in the $2 \% \mathrm{Si}$ specimens, the grain

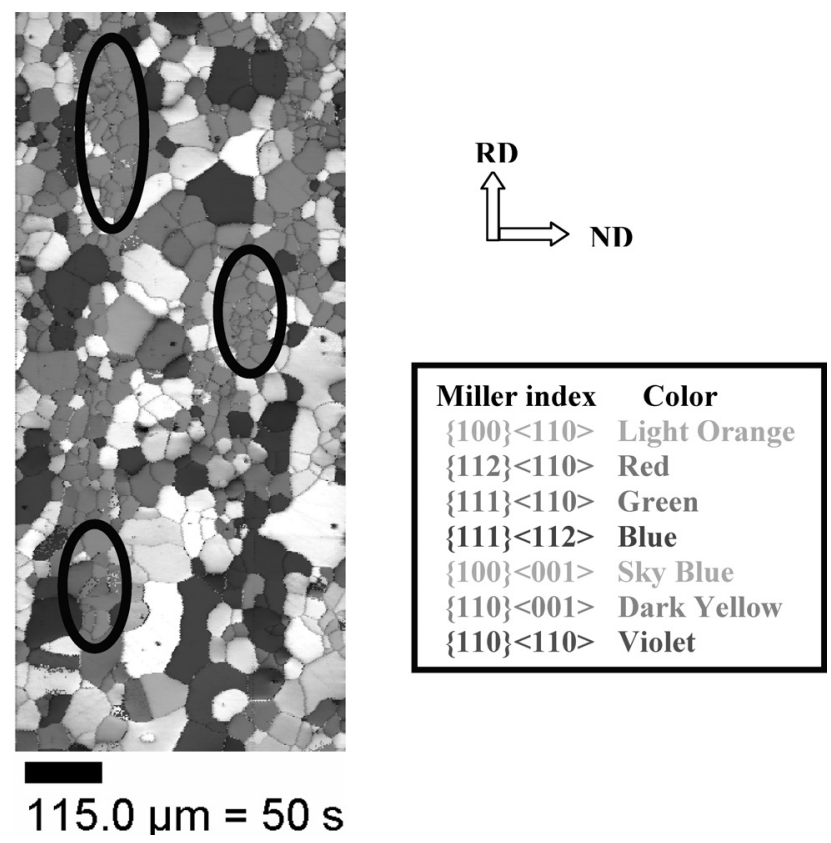

Fig. 6. An EBSD map coded with crystal orientation which shows grains of Goss orientation in colonies in the specimen annealed at $850^{\circ} \mathrm{C}$ (deviation angle: $20^{\circ}$ ).

size distribution is uniform throughout the thickness. Even in the specimen annealed at $740^{\circ} \mathrm{C}$ where grain growth process is slow, the grain size is relatively uniform throughout the thickness.

In order to observe texture inhomogeneity throughout the thickness, X-ray texture was measured at different layers defined as parameter $S$ for the specimen annealed at $740^{\circ} \mathrm{C}$. Figure 8 shows the textures presented in the $\varphi_{2}=45^{\circ} \mathrm{sec}-$ tion of ODF at $S=0.95,0.75,0.5$ and 0 respectively, in the specimen annealed at $740^{\circ} \mathrm{C}$. Although their domonant textures are broad $\{111\}\langle 112\rangle$ throughout the thickness, their maximum densities are quite different. Therefore, it seems reasonable to measure texture on a cross section in order to investigate texture development during grain growth. Figure 9 represents the changes in the area fraction of various texture components obtained from EBSD measurements as a function of annealing temperature. Two major texture components during the grain growth stage are $\{111\}\langle 112\rangle$ and $\{111\}\langle 110\rangle$. The area fraction of the $\{111\}\langle 112\rangle$ texture component increases significantly as the annealing temperature increases. The area fraction of the $\{111\}\langle 110\rangle$ texture component increases very slightly. On the other hand, the area fraction of the $\{001\}\langle 100\rangle$ texture component continues to decrease as the annealing temperature increases. The area fractions of other texture components remain almost constant or tend to decrease slightly during the grain growth stage. Based on these observations, we can deduce that both $\{111\}\langle 112\rangle$ and $\{111\}\langle 110\rangle$ texture components are strengthened mainly at the expense of random orientation during grain growth.

The average grain diameter of selected grain orientations was obtained using the OIM software. Total grain numbers used for this calculation were as follows: 2879 grains at $740^{\circ} \mathrm{C}, 2509$ grains at $790^{\circ} \mathrm{C}, 1478$ grains at $850^{\circ} \mathrm{C}$ and 1082 grains at $900^{\circ} \mathrm{C}$. The change in average grain diameter having $\{111\}\langle 112\rangle,\{111\}\langle 110\rangle,\{001\}\langle 100\rangle$ and ran- 


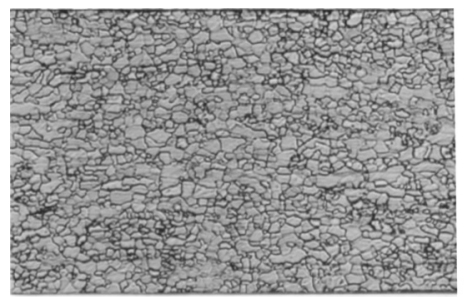

$740^{\circ} \mathrm{C}$

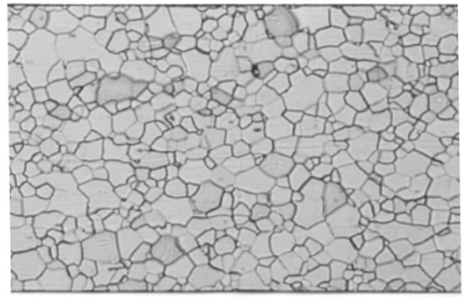

$850^{\circ} \mathrm{C}$

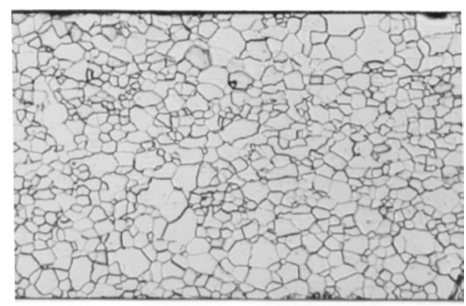

$790^{\circ} \mathrm{C}$

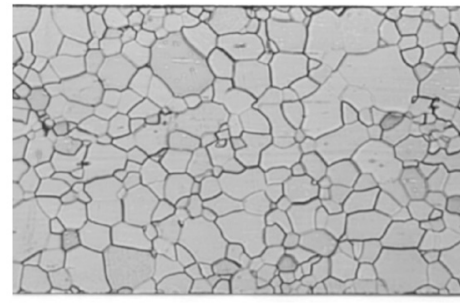

$900^{\circ} \mathrm{C} \quad \underline{100 \mu \mathrm{m}}$

Fig. 7. Optical microstructures at annealing temperature in the $1 \% \mathrm{Si}$ specimens.

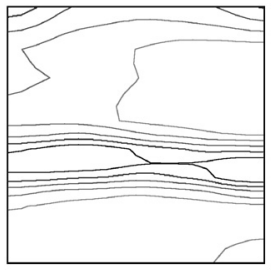

(a) $\mathrm{S}=\mathbf{0 . 9 5}$
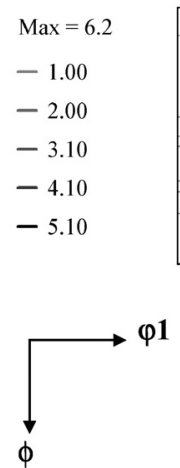

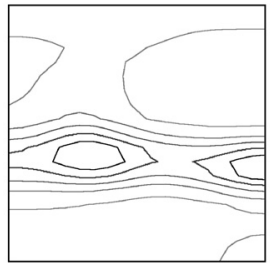

(c) $\mathrm{S}=0.5$

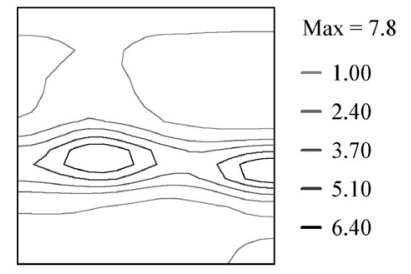

(b) $\mathrm{S}=\mathbf{0 . 7 5}$ $\varphi 1$

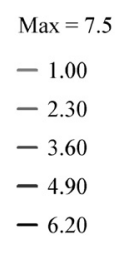

4.90

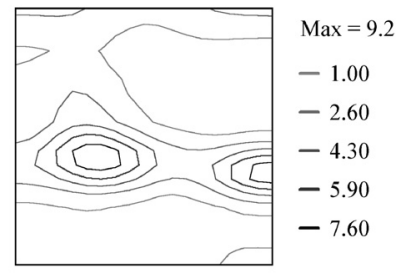

(d) $\mathrm{S}=\mathbf{0}$
Fig. 8. $\varphi_{2}=45^{\circ}$ sections of ODF at different layers in the $1 \% \mathrm{Si}$ specimen annealed at $740^{\circ} \mathrm{C}$.

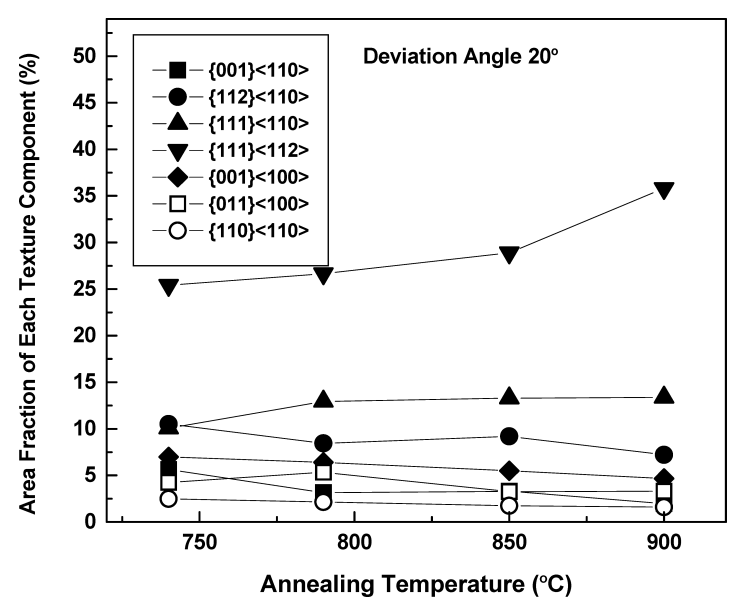

Fig. 9. Area fraction of grains with various orientations with annealing temperature in the $1 \% \mathrm{Si}$ specimens.

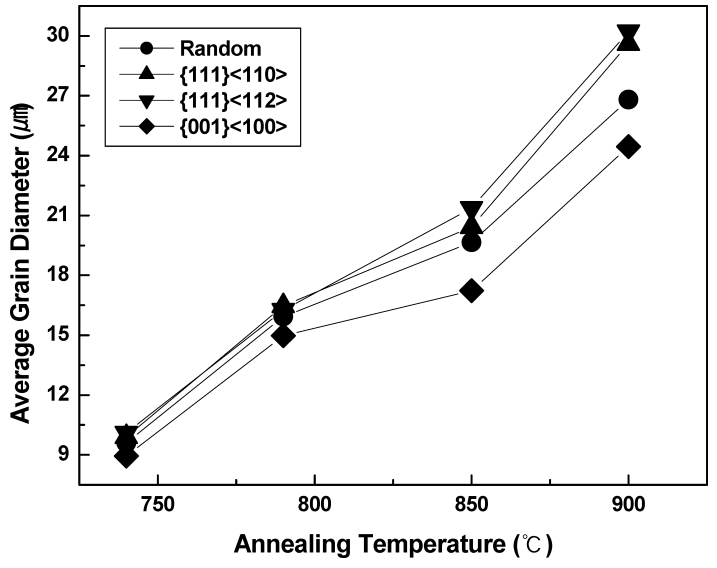

Fig. 10. Average grain diameter of grains with various orientations with annealing temperature in the $1 \% \mathrm{Si}$ specimens.

dom orientation with annealing temperature is shown in Fig. 10. The average grain diameter is different for these four orientations. The average diameter of grains having $\{111\}\langle 112\rangle$ and $\{111\}\langle 110\rangle$ orientation is bigger than that of grains having $\{001\}\langle 100\rangle$ and random orientation at each annealing temperature. Different sizes of grains of specific orientations may arise as a consequence of different growth rates for different orientations. Therefore, the grains of both $\{111\}\langle 112\rangle$ and $\{111\}\langle 110\rangle$ orientations may be expected to grow faster than those of $\{001\}\langle 100\rangle$ and random orientation during the grain growth stage.

We need to investigate why the grains of both $\{111\}\langle 112\rangle$ and $\{111\}\langle 110\rangle$ orientations in $1 \% \mathrm{Si}$ specimens increase in strength during grain growth. Correlated misorientation distribution for the grains of these orientations was obtained from EBSD measurements. The number of grain boundaries used for this analysis was 729, 391 and 152 for $\{111\}\langle 112\rangle,\{111\}\langle 110\rangle$ and $\{001\}\langle 100\rangle$ grains, respectively. Figure 11 presents the misorientation angle distribution for the grains having these three orientations in the specimen annealed at $740^{\circ} \mathrm{C}$. The $\{001\}\langle 100\rangle$ grains have the highest frequency of low angle grain boundaries 


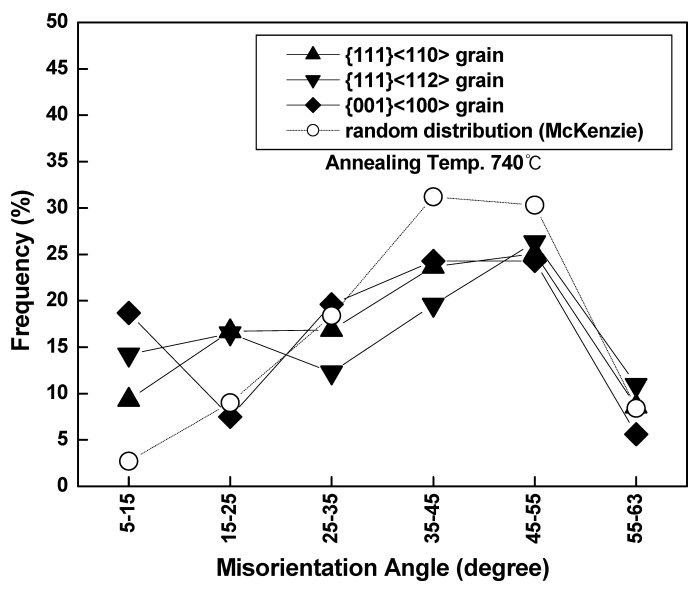

Fig. 11. Correlated misorientation angle distribution of grains with $\{111\}\langle 112\rangle,\{111\}\langle 110\rangle$ and $\{001\}\langle 100\rangle$ orientations in the $1 \% \mathrm{Si}$ specimen annealed at $740^{\circ} \mathrm{C}$.

(less than $15^{\circ}$ misorientation). On the other hand, $\{111\}\langle 112\rangle$ and $\{111\}\langle 110\rangle$ grains show a higher frequency of high angle grain boundaries than $\{001\}\langle 100\rangle$ grains. It is generally known that the mobility of high angle grain boundaries is significantly higher than that of low angle grain boundaries ${ }^{10-12)}$ and the boundary energy of high angle grain boundaries is also higher than that of low angle grain boundaries. ${ }^{13)}$ In addition, Hayakawa et al. ${ }^{14,15)}$ and Rajmohan et al. ${ }^{16,17)}$ assumed that the mobility of high energy grain boundaries in $\mathrm{Fe}-\mathrm{Si}$ steels is higher than that of low energy grain boundaries and this difference could be explained using the vacancy mechanism. It is well known that the migration of grain boundaries takes place by the exchange of atoms between grains through vacancies. ${ }^{18-20)}$ The high energy grain boundaries are more capable of absorbing and emitting vacancies than the low energy grain boundaries ${ }^{21)}$ because the number of vacancies is closely related to disorder in grain boundary structure. The high energy grain boundaries have a more disordered structure and hence a high number of vacancies and dislocations contributes to a high mobility. Therefore, the grains with high angle, high energy grain boundaries are expected to grow, whereas grains surrounded by low angle, low energy grain boundaries are not expected to continue to grow. As a result, the grains of $\{111\}\langle 112\rangle$ and $\{111\}\langle 110\rangle$ orientations have a high probability of consuming the grains of $\{001\}\langle 100\rangle$ and random orientation. From a comparison between Figs. 5 and 11, it should be noted that $\{111\}\langle 112\rangle$ and $\{111\}\langle 110\rangle$ orientations in the $1 \%$ Si specimens have a higher frequency of high angle, high energy grain boundaries (higher than $15^{\circ}$ misorientation) than Goss and $\{111\}\langle 112\rangle$ grains in the $2 \% \mathrm{Si}$ specimens. On the other hand, in the $1 \% \mathrm{Si}$ specimens, $\{111\}\langle 112\rangle$ grains have a higher frequency of low angle grain boundaries than $\{111\}\langle 110\rangle$ grains. However, this higher frequency of low angle grain boundaries in $\{111\}\langle 112\rangle$ grains does not seem to play a role in inhibiting grain growth because the $\{111\}\langle 112\rangle$ grains are uniformly distributed and do not form colonies like the Goss grains in Fig. 6. This fact was confirmed by EBSD measurements. At the beginning of grain growth, the two strongest texture components, $\{111\}\langle 112\rangle$ and $\{111\}\langle 110\rangle$, have been strengthened main- ly at the expense of the grains of random orientation as the annealing temperature increases. The grains of these orientations have also a size advantage over those of random orientation. In addition, the grains of $\{111\}\langle 112\rangle$ and $\{111\}\langle 110\rangle$ orientations have a more favorable grain boundary character distribution for further growth than those of $\{001\}\langle 100\rangle$ orientation. Because of these two reasons, both $\{111\}\langle 112\rangle$ and $\{111\}\langle 110\rangle$ texture components are strengthened during the grain growth stage.

As stated above, there are two different results concerning texture development during grain growth. In the $2 \% \mathrm{Si}$ specimens, the major texture components such as the Goss and $\{111\}\langle 112\rangle$ components are reduced in strength. The grains of these orientations have on average a smaller size than those of random orientation. In contrast, in the $1 \% \mathrm{Si}$ specimens, the major texture components such as the $\{111\}\langle 112\rangle$ and $\{111\}\langle 110\rangle$ components are strengthened at the expense of random orientation. The grains of $\{111\}\langle 112\rangle$ and $\{111\}\langle 110\rangle$ orientations have a size advantage over those of random orientation. However, it should be noted that the size advantage might not be a sufficient condition for texture strengthening during grain growth. We can easily infer from Figs. 5 and 6 that although some grains of specific orientations have a size advantage, the grains in colonies (i.e., having a very high frequency of low angle, low energy grain boundaries) cannot continue to grow faster and will ultimately be consumed. Therefore, we can claim that for a texture component to be continuously strengthened during grain growth, the grains of the specific orientation should have not only a size advantage over those of other orientations, but also a higher frequency of high angle, high energy grain boundaries.

\section{Conclusions}

In the $2 \% \mathrm{Si}$ specimens, the major texture components, Goss and $\{111\}\langle 112\rangle$ components, are weakened and the random texture is strengthened during grain growth. The grains of the Goss and $\{111\}\langle 112\rangle$ orientations have smaller grain size than those of random orientation. The Goss texture component continues to decrease in strength from the beginning of grain growth because the Goss grains have a high percentage of low angle, low mobility grain boundaries.

In the $1 \% \mathrm{Si}$ specimens, the main texture components, $\{111\}\langle 112\rangle$ and $\{111\}\langle 110\rangle$ components, are strengthened mainly at the expense of grains having random orientation. The grains of $\{111\}\langle 112\rangle$ and $\{111\}\langle 110\rangle$ orientations have a size advantage over the grains of random orientation. In addition, the grains of $\{111\}\langle 112\rangle$ and $\{111\}\langle 110\rangle$ orientations have a higher frequency of high angle, high energy grain boundaries than those of $\{001\}\langle 100\rangle$ orientation, a decreasing component.

Based on obtained experimental results, some general rules which govern texture development during grain growth in nonoriented electrical steels can be proposed. For a texture component to be strengthened during grain growth, the grains of the specific orientation should have not only a size advantage over those of other orientations, but also a higher frequency of high angle, high energy grain boundaries. This conclusion supports our model of grain 
growth in grain-oriented electrical steels described in previous papers. ${ }^{14-17)}$

\section{REFERENCES}

1) B. D. Cullity: Introduction to Magnetic Materials, Addison-Wesley Publishing Company, Reading, Mass., (1972), 502.

2) H. Shimanaka, Y. Ito, K. Matsumura and B. Hukuda: J. Magn. Magn. Mater, 26 (1982), 57.

3) J. T. Park, J. S. Woo and S. K. Chang: J. Magn. Magn. Mater, 182 (1998), 381

4) J. T. Park, R. E. Park and J. S. Woo: J. Korean Inst. Met. Mater, 32 (1994), 616.

5) J. A. Slane and P. A. Labun: Energy Efficient Electrical Steels, TMSAIME, Warrendale, PA, (1980), 169.

6) B. Hutchinson and E. Nes: Grain Growth in Polycrystalline Materials, Trans. Tech. Publications, Zurich, (1992), 385.

7) P. Balke: Ph. D. Thesis, University of Groningen, the Netherlands, (2002).

8) J. T. Park: Ph. D. Thesis, McGill University, Canada, (2002).

9) F. J. Humphreys and M. Hatherly: Recrystallization and Related An- nealing Phenomena, Elsevier Science Limited, Pergamon, Oxford, (1995), 304.

10) A. O. Humphreys and F. J. Humphreys: Proc. 4th Int. Conf. on Aluminium, Vol. 1, Georgia Institute of Technology, Atlanta, (1994), 211.

11) T. Furu: Ph. D. Thesis, NTH, Trondheim, (1992).

12) R. Viswanathan and C. L. Bauer: Acta Metall., 21 (1973), 1099.

13) G. C. Hasson and C. Goux: Scr. Metall., 5 (1971), 889.

14) Y. Hayakawa and J. A. Szpunar: Acta Mater, 45 (1997), 1285.

15) Y. Hayakawa and J. A. Szpunar: Acta Mater., 45 (1997), 4713.

16) N. Rajmohan, J. A. Szpunar and Y. Hayakawa: Textures Microstruct., 32 (1999), 153.

17) N. Rajmohan and J. A. Szpunar: Mater. Sci. Eng. A, A289 (2000), 99.

18) G. Gottstein and F. Schwarzer: Mater. Sci. Forum., 94-96 (1992), 187.

19) B. Ralph, K. B. Shim, Z. Hudd, J. Forley and M. Edirisinghe: Mater. Sci. Forum., 94-96 (1992), 129.

20) S. E. Babcock and R. W. Balluffi: Acta Metall., 37 (1989), 2367.

21) W. Hahn and H. Gleiter: Acta Metall., 29 (1980), 601. 